\title{
Familial Chylomicronaemia: A Neonate with Milky White Blood
}

Chaudhary NK', Chapagain $\mathrm{RH}^{2}$, Siapi S ${ }^{3}$, Chaudhary S ${ }^{4}$, Paudel KP5

\section{Abstract}

Familial Chylomicronaemia is a rare autosomal recessive disease of lipoprotein metabolism characterized by deficiency or absence of lipoprotein lipase (LPL) or its co-factor apoC-II which causes severe elevation of triglyceride and chylomicron resulting in lipaemic plasma, recurrent attacks of acute pancreatitis, eruptive xanthomas, hepato-splenomegaly and lipaemiaretinalis. We report a case of term female neonate with lipaemic plasma, lipemia retinalis, markedly elevated triglyceride level which is consistent with diagnosis of Familial Chylomicronaemia.

Key words: Familial Chylomicronaemia, lipaemic plasma, triglyceride level, Lipaemiaretinalis.

\section{Introduction}

$\mathrm{F}$ amilial Chylomicronaemia or previously called Type 1 Hyperlipidemia is a rare disease of lipoprotein metabolism with incidence of approximately 1:1000000 population ${ }^{1}$. This condition is characterized by deficiency or absence of lipoprotein lipase (LPL), or its co-factor apoC-II, which causes severe elevation of triglyceride rich plasma chylomicrons and is associated with decrease in HDL cholesterol $^{2}$. Both this condition is inherited as autosomal recessive disorder caused by mutation in genes that encode for key molecules in the lipolytic pathway. Almost all patients have serum triglyceride level in excess of $1000 \mathrm{mg} / \mathrm{dl}$ (11.36 mmoL/L), upto $10,000 \mathrm{mg} / \mathrm{dl}$. These patients usually have lipaemic plasma due to high level of triglyceride and circulating chylomicrons. It manifests as eruptive xanthoma, acute pancreatitis, hepatosplenomegaly, foam cell infiltration of bone marrow and lipaemiaretinalis. However cases remain clinically silent and undiagnosed until blood is sampled for another purpose ${ }^{3}$.

Here, we report a case of Familial Chylomicronaemia (Type I Hyperlipidaemia) diagnosed in a new-born infant as we didn't find any case report of Familial Chylomicronaemia diagnosed in new-born reported in Nepal so far.

\section{The Case}

A twenty eight days term female neonate born of nonconsanguineous marriage, birth weight of $2.7 \mathrm{~kg}$ with uneventful antenatal, natal and postnatal period who is exclusively breast fed
${ }^{1}$ Dr. Nanda Kishwor Chaudhary, (MBBS) MD Resident, ${ }^{2} \mathrm{Dr}$. Ram Hari Chapagain (MBBS, MD) Asst. Professor, Senior Consultant, ${ }^{3} \mathrm{Dr}$. Sani Sipai (MBBS) MD Resident, ${ }^{4} \mathrm{Dr}$. Suryakant Chaudhary (MBBS) MD Resident. ${ }^{5}$ Dr. Krishna Prasad Paudel (MBBS, MD) Associate Professor, Chief Consultant. All from the National Academy of Medical Sciences (NAMS), Kanti Children's Hospital, Kathmandu, Nepal.

\section{Address for correspondence \\ Dr. NK Chaudhary \\ E-mail:nandakishorchaudhary2015@gmail.com}

\section{How to cite}

Chaudhary NK, Chapagain RH, Siapi S, Chaudhary S, Paudel KP. Familial Chylomicronaemia: A Neonate with Milky White Blood. J Nepal Paediatr Soc 2018;38(2):128131.

doi: http://dx.doi.org/10.3126/jnps.v38i2.20473

This work is licensed under a Creative Commons Attribution 3.0 License.

\section{(c) (i)}


and having adequate weight gain(present weight $4 \mathrm{~kg}$ ) presented in emergency department of Kanti Children's Hospital, Kathmandu, Nepal with complains of fever and cough since last one day. The neonate was admitted in emergency ward with clinical diagnosis of late onset neonatal sepsis.

While being investigated for sepsis, blood sample was found to be lactescent (Figure1).There was no family history of hyperlipidaemia syndromes, recurrent pancreatitis, xanthomas and early cardiovascular disease.

On ophthalmologic examination of the neonate, she was found to have abnormal lipid deposited on retina (lipaemiaretinalis). On overnight refrigeration of centrifuged blood sample, creamy white supernatant was found to be floating at the top of sample (Fig II). Next day, blood sample was collected, which too was milky white and investigations done showed Triglyceride: $13812 \mathrm{mg} / \mathrm{dl}$, Cholesterol $1708 \mathrm{mg} / \mathrm{dl}$, HDL cholesterol: $8 \mathrm{mg} / \mathrm{dl}$, LDL cholesterol: could not be calculated, Haemoglobin: $12.8 \mathrm{gm} / \mathrm{dl}$, PCV: 23.1\%,Total leukocyte count: 16,500/cumm, with Polymorph 33\%, Lymphocyte $60 \%$, Monocyte $04 \%$ and eosinophil 03\%, Platelet count: $3,45,000 /$ cumm, Total serum bilirubin: 0.5 mg/dL, C-reactive Protein: Negative, RBS: $126 \mathrm{mg} / \mathrm{dL}$ (glucometer strip), TSH: $1.24 \mu \mathrm{lU} / \mathrm{ml}$, Free T3: $4.89 \mathrm{pg} / \mathrm{ml}$ and Free T4: $1.65 \mathrm{ng} / \mathrm{dL}$. Ultra sonographic examination of abdomen and pelvis revealedd hepatomegaly.

Other secondary causes of hyperchylomicronaemia are hypothyroidism, nephritic syndrome, diabetes mellitus, congenital biliary atresia, excessive alcohol intake and oral intake oral contraceptive pills and thiazide diuretics. Hypothyroidism which was excluded by normal thyroid function test results, nephritic syndrome was ruled out by normal urine routine examination reports, congenital biliary atresia was ruled out by ultrasonography of abdomen, diabetes mellitus was excluded by normal blood glucose level and use of drugs and alcohol were excluded by history. Other condition with mixed dyslipidaemia (plasma triglyceride level between 200 and $800 \mathrm{mg} / \mathrm{dl}$, plasma cholesterol level between 200 and $400 \mathrm{mg} / \mathrm{dl}$ and HDL-C level less than $40 \mathrm{mg} / \mathrm{dl}$ is seen in familial combined hyperlipidaemia, which being autosomal dominant condition has typical family history of premature cardiovascular disease and triglyceride and cholesterol level are usually moderately elevated with clear plasma appearance however in this case there is marked elevation of triglyceride and cholesterol level with Total triglyceride (TG):Total cholesterol (TC) ratio of 8.08 (ratio of $>5$ is highly suggestive of circulating chylomicron) and milky white plasma.
On this ground, diagnosis of Type 1 Hyperlipidemia or Familial chylomicronaemia was made. New-born was managed with intravenous antibiotics for neonatal sepsis and parents were counselled for fat restriction and was prescribed medium chain triglyceride (medium chain triglycerides are directly adsorbed into the portal venous system and may augment total fat intake) and fat soluble vitamins. Child was discharged after five days with oral antibiotics, fat soluble vitamins and medium chain triglycerides.

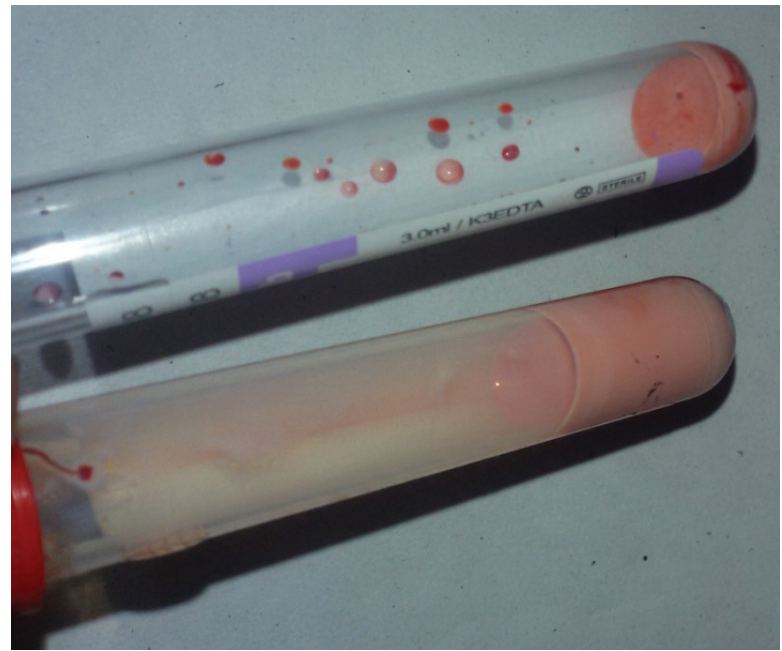

Fig 1: Lactescent blood sample.

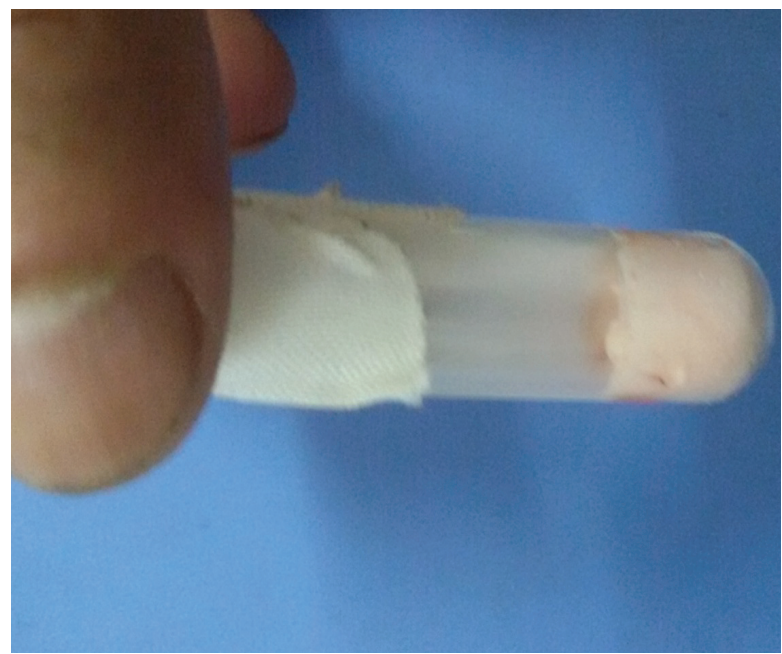

Fig 2: Creamy white supernatant after overnight refrigeration of the centrifuged blood sample.

\section{Discussion}

Familial Chylomicronaemia is a rare metabolic disorder which is inherited as autosomal recessive disorder. It is characterized by severe fasting hypertriglyceridaemia and chylomicronaemia in plasma resulting from LPL or apoprotein C-II deficiency². The hallmark of disease is abnormal persistence of circulating chylomicron in blood following fasting period 
of 12-14 hour ${ }^{4}$. Mutation in lipoprotein lipase (LPL) gene has been found in $80 \%$ of the cases reported in literature, and more than 180 mutations have been identified ${ }^{5}$.

It is characterised by markedly elevated triglyceride and circulating chylomicron resulting in lipaemic serum and recurrent episodes of abdominal pain owing to acute pancreatitis, eruptive xanthoma, hepatosplenomegaly and lipaemiaretinalis. Most cases of familial LPL deficiency usually present at age of 10 years, but $25 \%$ of the cases can present during infancy ${ }^{6}$, as our case, which was diagnosed at 28 days of life. Evidence suggests that presentation during infancy is heterogeneous and may present with signs such as pallor, jaundice, irritability and diarrhoea. These manifestations are variable in the time and severity of presentation ${ }^{7}$. Several cases have been reported from India in infants aged between 20 and 60 days and some presented with features of sepsis with systemic complications and renal failure with complete recovery ${ }^{8}$. Our case also presented with features of neonatal sepsis and the disorder was suspected only after obtaining blood sample for septic screening which was lipaemic. Electrophoresis of blood and genetic diagnosis of familial chylomicronaemia can be done but it is available only at limited centre so usually diagnosis is made clinically. In our case, diagnosis was made clinically on the basis of abnormal lipid profile results and lipaemiaretinalis on funduscopic evaluation of child, as genetic testing and electrophoresis is not available in our country and parents were not in position to afford for other investigations and the diagnosis was made by Kursid et al. in 10 days old in same way who presented with refusal to feed and irritability for last 24 hours $^{9}$.

Adenwala et al. reported a case of Familial Chylomicronaemia Syndrome in a five months infants with Cleft palate. They found milky blood per-operatively while giving incision for repairing cleft palate ${ }^{3}$.

Borgheiet al. reported a case of Familial Chylomicronaemia Syndrome in a five months old infant presenting with acute necrotizing pancreatitis. In this case the neonate was born of consanguineous marriage ${ }^{10}$, but in our case, there was no history of consanguineous marriage and no history of acute pancreatitis, early cardiovascular disease and eruptive xanthoma in parents and sibling.

Eruptive xanthoma and lipaemiaretinalis may be usually present when plasma triglyceride concentration exceeds $2000 \mathrm{mg} / \mathrm{dl}$ and $4000 \mathrm{mg} / \mathrm{dl}$ respectively. Xanthomas are painless skin lesions present on back and buttocks and extensor surface of arms and legs. The acuteness of symptoms are directly proportional to the level of chylomicronaemia. Chylomicronaemia cause neurological manifestation and dyspnoea. Hepatosplenomegaly results due to accumulation of triglycerides in the reticuloendothelial system ${ }^{3}$.In our case only lipaemiaretinalis and hepatomegaly was present but no eruptive xanthoma and splenomegaly were present.

LPL deficiency is suspected when serum is lipaemic after 12 hours of fasting and fasting plasma is turbid and if left at $4^{\star} \mathrm{C}$ for few hours, chylomicrons appear at the top forming creamy supernatant ${ }^{3}$. In our case also creamy white supernatant was found at the top of plasma after overnight refrigeration.

The diagnosis of Chylomicronaemia is made by accessing LPL activity in plasma after intravenous administration of heparin. Heparin binds with LPL causing its dissociation from heparin sulphate from endothelial surface with its subsequent release into plasma ${ }^{3}$.When LPL deficiency is suspected, genetic analysis is needed to confirm the diagnosis. The full gene sequencing of LPL and four other proteins is gold standard to precisely determine which mutated protein forms basis for this metabolic disorder ${ }^{4}$. Diagnosis of Apo C-II deficiency is made by demonstration of low LPL activity in postheparin plasma and restoration of normal enzymatic activity on addition of Apo C-II to the assay mixture. Another diagnostic method is immunoassay of Apo C- $\mathrm{II}^{3}$. However these investigations were not performed in our case due to lack of these facilities in Nepal and low socioeconomic condition of parents.

There is no pharmacological agent to treat this condition till date. The principle modality of management is severe dietary triglyceride restriction. The fat should be restricted to less than $20 \mathrm{gm} /$ day and less than $15 \%$ of total caloric intake so that triglyceride is restricted below $1500 \mathrm{mg} /$ day to maintain serum triglyceride level below $16.94 \mathrm{mmol} / \mathrm{L}$. Preferred source of fat should be medium chain triglyceride which should not exceed 0.5 $\mathrm{gm} / \mathrm{kg} /$ day. With these dietary modification for long time, abdominal pain will be relieved or even disappear and liver and spleen size becomes normal. Coronary artery disease will not develop as complication and child can live as long as others ${ }^{6}$. Our case was also advised for fat restricted milk, medium chain triglyceride and fat soluble vitamins and child was found to be asymptomatic and doing well on follow up till two and half months of age.

\section{Conclusion}

Familial chylomicronaemia is a rare disease usually diagnosed incidentally with clinical and limited laboratory ground and can be diagnosed on neonatal period. Dietary management is the main stay of therapy. 


\section{References}

1. Mohandas MK, Jemila J, Ajith Krishnan AS, George TT. Familial Chylomicronemia syndrome. Indian J Pediatr.( Internet). 2005 Feb (cited 2018 Nov 18): 72(2):181. Available from: https://www.ncbi.nlm.nih. gov/pubmed/15758547

2. Stanley AC, Bennett MJ. Defects in Metabolism of lipids. In: Kleigeman RM, Stanton BF, III JWSG, Scholar NF, Behrman RE, editors. Nelson Text Book of Pedaitrics. $20^{\text {th }}$ Ed. Elseveir. 2016. p78-715.

3. Adenwalla HS, Narayanan PV, Rajshree CJ, Santhakumar R. An Interesting case of Familial Chylomicronemia Syndrome in a cleft palate child. Indian J Plast Surg 2008; 41(1)70-2. DOI: 10.4103/0970-0358.41116

4. Brahm AJ, Hegele R. Chylomicronemia current diagnosis and future therapies. Nat Rev Endocrinol 2015;11(6):352e62 DOI: 10.1038/nrendo.2015.26

5. Rabacchi C, Pisciotta L, Cafalu AB, et al . Spectrum of the LPL gene identified in Italy in patients with severe hypertriglyceridemia Atherosclerosis 2015;241(1):7986 DOI: 10.1016/j.atherosclerosis.2015.04.815
6. Sugandhan S, Khandpur S, Sharma VK. Familial Chylomicronemia Syndrome. Pediatr Dermatol 2007;24(3):323-25. DOI: 10.1111/j.15251470.2007.00415.x

7. Rahalkar AR, Hegele RA. Monogenic pediatric dyslipidemias: classification, genetics and clinical spectrum. Mol Genet Metab 2008;93(3):282-9 DOI: 10.1016/j.ymgme.2007.10.007

8. Nagar R, Arora U.An unusual case of familial hyperlipidaemia. Indian Journal of Clinical Biochemistry. 2008;23(3): 302-304 (Cited 2018 Nov 18) Accessed from https://link.springer.com/article/10.1007/s12291008-0069-4

9. Wani K, Rashid M, Alaqaband MM, Manzoor S, Mushtaq S. Familial Chylomicronemia Syndrome in a 10-day-old Neonate: A Case Report. Int J Pediatr. 2015;3(N.1-2,Serial N 14): 449-453 (Cited 2018 Nov 18) Available from https://www.researchgate.net/ publication/271326631_httpijpmumsacirarticle_3866_ Ohtml

10. BorgheiA,Azizi M. Familial Chylomicronemia Syndrome presenting with Acute Necrotising Pancreatitis in a Five Month Infant. J Nepal Paediatr Soc 2010;30(2):110-2 doi:10.1097/MPA.0000000000000811 